\title{
Current-Density Functional Theory of the Response of Solids
}

\author{
Neepa T. Maitra, Ivo Souza, and Kieron Burke \\ Dept of Chemistry and Chemical Biology and Dept of Physics and Astronomy, Rutgers University, Piscataway, NJ 08854
}

(Dated: submitted: May 8, 2002; revised November 6, 2018)

\begin{abstract}
The response of an extended periodic system to a homogeneous field (of wave-vector $q=0$ ) cannot be obtained from a $q=0$ time-dependent density functional theory (TDDFT) calculation, because the Runge-Gross theorem does not apply. Time-dependent current-density functional theory is needed and demonstrates that one key ingredient missing from TDDFT is the macroscopic current. In the low-frequency limit, in certain cases, density polarization functional theory is recovered and a formally exact expression for the polarization functional is given.
\end{abstract}

PACS numbers: 71.15.Mb,78.20.-e,78.20.Ci,73.63.-b

Density functional theory [1, 2] is a standard approach for calculating ground-state properties of solids [3] and molecules [4]. Time-dependent density functional theory (TDDFT) is an extension of the ground-state formalism based on the Runge-Gross theorem [5]; this establishes a one-to-one correspondence between time-dependent densities and time-dependent one-body potentials. When a time-dependent electric field is applied to a system, this formalism provides a route to its optical response 6 . The response equations of TDDFT have been encoded in standard quantum chemical packages 7], and results for molecules are appearing (see Ref. [8] for many examples). As in the ground-state case, the accuracy depends on the quality of the approximate functional used.

There is great interest in applying the same technique to extended systems. While these can be treated well within existing wavefunction technology, using, e.g., the GW approximation and then solving the BetheSalpeter equation for the optical response 9 , the allure of a TDDFT approach is its far lower computational cost. Calculations already show that excitonic effects appear to be treatable by going beyond the usual local and semi-local approximations of standard DFT calculations [10, 11].

There is also a version of the time-dependent theory, called time-dependent current-density functional theory (TDCDFT), that uses the current-density as the basic variable: As the choice of variable (charge density versus current density) appears a matter of convenience, TDCDFT and TDDFT appear to be equivalent (for non-magnetic systems). The time-dependent exchange-correlation potential has been argued to be more amenable to local and semilocal approximation in terms of the current-density [12] and this framework has been used in recent response calculations of solids [13, 14] and conjugated polymers [15]. Initial work towards a matrix formulation of the current-density response equations has been presented in Ref. 16].

In this paper, we demonstrate a difference in principle between the two approaches when applied to bulk solids. The basic theorems of DFT, ground-state or timedependent, are proven for finite electronic systems (i.e. systems with a boundary). We consider the response of periodic systems (such as the bulk of an insulator or metal) to time-varying electric fields which have a spatially uniform component. We show that TDDFT fails in this case: there is no one-to-one correspondence between the time-varying periodic density and the applied potential. Instead, TDCDFT is needed for a complete description. The time-dependent potential and all response properties are not functionals of the time-dependent periodic density, but rather are functionals of the timedependent periodic current density. In the limit of low frequencies our analysis recovers the well-known GGG theorem [17, if microscopic transverse currents can be neglected; TDCDFT then recovers (static) density polarization functional theory [17, 18].

Modern solid-state calculations model extended periodic systems and extract bulk properties by using periodic boundary conditions. Our first point is that the Runge-Gross (RG) theorem does not apply when a homogeneous electric field is applied to a periodic system. RG states that, given an initial state, there is a oneto-one correspondence between time-dependent densities and time-dependent scalar potentials for a given interaction and statistics. The first step of the RG proof establishes a one-to-one correspondence between potentials and currents [20]. In the second step, continuity is then used to relate currents to densities, and a oneto-one mapping between densities and potentials results provided a certain surface integral involving the density and the potential vanishes. For finite physical systems the condition for requiring this surface term to be zero can be given rigorously for systems in which the density vanishes at the surface 21]. For a periodic system, one might try to choose a surface around which the density and potential are periodic but for a uniform field the linearity of the potential prevents this, and TDDFT does not apply.

Another way to see this is in modelling the periodic system in an electric field by a large ring of length $L$ of the material, through which is threaded a time-varying solenoidal magnetic field [19]. This field produces a uniform vector potential on the system, $\mathbf{A}(t)$, that corresponds to a macroscopic electric field along the ring. The beauty of this appoach is that the Hamiltonian remains spatially periodic, although it becomes time-dependent. TDDFT was however derived with only scalar potentials 
in mind, and does not consider such transverse vector potentials; such uniform electric fields cannot be generated by a charge distribution. The first part of RG still holds in this case however, showing the potential is a unique functional of the current-density.

Thus the density in the interior of any system is insufficient information to deduce the external electric field, but the current-density is sufficient. This is a time-dependent generalization of the original GGG theorem [17]. We shall come back to the static case shortly.

A simple example demonstrating the non-uniqueness of the potential-density mapping is a noninteracting free electron gas on a ring, subjected to a constant, uniform electric field, $\mathcal{E}$, turned on at time $t=0$. Representing the field by a vector potential $A=-c \mathcal{E} t$, each orbital in the Slater determinant state satisfies the time-dependent Schrödinger equation

$$
(\hat{p}-\mathcal{E} t)^{2} \phi_{m} / 2=i \dot{\phi}_{m}
$$

(We use atomic units throughout this paper). If the electrons are initially in an eigenstate, $\phi_{m}(x, 0)=$ $e^{i 2 \pi m x / L} / \sqrt{L}$, where $x$ is the coordinate around the ring, and the conjugate momentum is $k_{m}=2 \pi m / L$, with $m$ an integer between 0 and $L$, different for each orbital, then the solution at time $t$ is readily found to be

$$
\phi_{m}(x, t)=e^{-i\left(k_{m}^{2} t / 2-k_{m} \mathcal{E} t^{2} / 2+\mathcal{E}^{2} t^{3} / 6\right)} e^{i 2 \pi m x / L} / \sqrt{L} .
$$

Since the electric field only affects the phase of this orbital, its density, and that of the noninteracting gas, $n(x, t)=\sum_{m=1}^{N}\left|\phi_{m}(x, t)\right|^{2}$, remains spatially uniform forever. In particular, two different electric fields give rise to exactly the same time-(in)dependent density. Thus the external potential is not uniquely determined by the density here. (This argument holds for any number $N$ of electrons).

The first part of $\mathrm{RG}$ remains valid 20] and applies to arbitrary vector potentials, not just those describing an electric field. Choosing a gauge in which the scalar potential vanishes, one can show there is a one-to-one correspondence between $\mathbf{A}(\mathbf{r} t)$ and $\mathbf{j}(\mathbf{r} t)$ for a given initial state 22, 23, 24] and this provides the formal basis for TDCDFT. In this gauge all electric fields are represented by vector potentials but the one-to-one correspondence is of course gauge-independent. In our simple example, the physical current density is given by $j(t)=\sum_{m}^{N} k_{m}+N \mathcal{E} t / L$. In two different electric fields, two different currents arise. More generally, for a periodic potential on a (1-dimensional) ring, when a uniform electric field is turned on, the density, current, etc., remain periodic, and each can be written as $\sum j_{G} \exp (i G x)$, where $G=2 n \pi / a$, and $n$ is an integer. All components of the current-density at $G \neq 0$ are determined by the periodic density by the continuity equation:

$$
j_{G}(\omega)=\omega n_{G}(\omega) / G, \quad(G \neq 0) .
$$

When a uniform electric field is present, the $G=0$ component (the macroscopic current) is undetermined by the time-dependent periodic density.
When the wavelength of the external field is finite, $\mathrm{RG}$ does apply to the periodic system; the macroscopic current is a functional of the periodic density (although cannot be determined by Eq. (3)). Examining the second step in the RG proof in one-dimension, one may choose the surface to be two points separated by an integral multiple of the lattice constant and the wavelength of the external field: the Hamiltonian is periodic around this surface, and the integrand of the surface term is the same at the two points. Thus the surface term vanishes and the $\mathrm{RG}$ theorem holds. This is consistent with the groundstate case [18] where the macroscopic polarization is only an independent variable when the external field has a homogeneous component. In our simple example, consider applying a perturbative electric field $\mathcal{E} \cos (q x)$ around the ring, where a finite number of wavelengths fits into the ring so $q$ is an integer multiple of $2 \pi / L$. The density picks up a spatial modulation, which, in the limit that $q \rightarrow 0$, becomes, to first order in $\mathcal{E}$,

$$
n(x t) \rightarrow N\left(1+\mathcal{E} q \sum_{m=1}^{N}\left(x+k_{m} t\right)^{3} / 3\right) / L .
$$

Different fields yield different densities except when $q=$ 0 , consistent with the 1-1 mapping between densities and potentials at finite wavelengths. One can then imagine attempting to find the uniform-field value of certain response functions 25] by taking the $q \rightarrow 0$ limit of a series of finite- $q$ TDDFT calculations, although the larger supercell required might render this procedure impractical (although, see discussion of Ref. [10] below). The same issue arises in the ground-state case, where it was shown in Ref. 26] how careful use of a sawtooth potential on a supercell can resolve this problem. The approximation for the density-functional must be ultra-nonlocal in space in order to capture the exchange-correlation fields generated from the charge distribution modulated by the long wavelength. The first excitonic peak in the dielectric function of silicon (missing when using any local or semi-local approximation in TDDFT) was captured in Ref. 11] in a TDDFT calculation at finite wavevector, using an ultranonlocal kernel $f_{\mathrm{XC}} \sim \alpha / q^{2}$ however with $\alpha$ being empirically determined, and with applying a GW shift to the spectrum. In Ref. [10] an exact exchange calculation was performed, reproducing the excitonic peak of silicon, without having to battle through a monstrously large numerical computation. The authors were able to analytically extract the diverging ultranonlocal factor $1 / q^{2}$ from the exact-exchange kernel and so needed to numerically evaluate only the remaining matrix elements, well-behaved as a function of $q$ : because the long-range behaviour had been already factored out, the numerical computation converged rapidly with the number of $k$-points.

However, like density-polarization functional theory in the ground-state case, TDCDFT allows one to obtain the homogeneous field result directly from a single $q=0$ calculation; this cannot be done in TDDFT. 
Having established that the current is needed when calculating bulk response properties in solids in uniform fields, we next review how such a calculation is performed for any electronic system. The one-to-one correspondence between $\mathbf{A}(\mathbf{r} t)$ and $\mathbf{j}(\mathbf{r} t)$ can be used to establish a set of KS equations in which noninteracting electrons move in a KS vector potential $\mathbf{A}_{\mathrm{S}}(\mathbf{r} t)$ and reproduce the exact current-density $\mathbf{j}(\mathbf{r} t)$. Assume the exact KS ground-state has been found and is nondegenerate. The TDCDFT KS equations are

$$
\left\{\frac{1}{2}\left[\mathbf{p}+\frac{1}{c} \mathbf{A}_{\mathrm{S}}[\mathbf{j}](\mathbf{r} t)\right]^{2}+v_{\mathrm{S}}(\mathbf{r})\right\} \phi_{i}(\mathbf{r} t)=i \frac{\partial \phi_{i}(\mathbf{r} t)}{\partial t}
$$

where $v_{\mathrm{S}}(\mathbf{r})$ is the periodic ground-state KS potential and all time-dependence is in the $\mathrm{KS}$ vector potential $\mathbf{A}_{\mathrm{S}}[\mathbf{j}](\mathbf{r} t)$. The orbitals begin as the occupied groundstate KS orbitals. The KS vector potential is defined to produce the exact physical current density

$$
\mathbf{j}(\mathbf{r} t)=\Im \sum_{i o c c} \phi_{i}^{*}(\mathbf{r} t) \nabla \phi_{i}(\mathbf{r} t)-\frac{1}{c} n(\mathbf{r} t) \mathbf{A}_{\mathrm{S}}(\mathbf{r} t) .
$$

Departing from convention, we write

$$
\mathbf{A}_{\mathrm{S}}(\mathbf{r} t)=\mathbf{A}_{\mathrm{ext}}(\mathbf{r} t)+\mathbf{A}_{\mathrm{EM}}(\mathbf{r} t)+\mathbf{A}_{\mathrm{XC}}(\mathbf{r} t) .
$$

Here $\mathbf{A}_{\mathrm{EM}}(\mathbf{r} t)=\mathbf{A}_{\mathrm{H}}(\mathbf{r} t)+\mathbf{A}_{\mathrm{T}}(\mathbf{r} t)$ is the full electromagnetic potential, satisfying Maxwell's equation:

$$
\left\{\nabla^{2}-\frac{1}{c^{2}} \frac{\partial^{2}}{\partial t^{2}}\right\} \mathbf{A}_{\mathrm{EM}}-\nabla \cdot\left(\nabla \cdot \mathbf{A}_{\mathrm{EM}}\right)=-\frac{4 \pi}{c} \mathbf{j} .
$$

The longitudinal component, $\mathbf{A}_{\mathrm{H}}$, is the vector equivalent of the Hartree potential, while the transverse component, $\mathbf{A}_{\mathrm{T}}$, arises from the transverse component of the current:

$$
\mathbf{A}_{\mathrm{T}}(\mathbf{r} t)=\frac{1}{c} \int d^{3} r^{\prime} \frac{\mathbf{j}_{\mathrm{T}}\left(\mathbf{r}, t-\left|\mathbf{r}-\mathbf{r}^{\prime}\right| / c\right)}{\left|\mathbf{r}-\mathbf{r}^{\prime}\right|} .
$$

In previous formulations of TDCDFT (e.g., Ref. 12]), this term does not appear explicitly. Since $\mathbf{A}_{\mathrm{T}}$ is a nonlocal classical electromagnetic contribution of any moving charge density, it is not an exchange-correlation effect, and should be included exactly in any time-dependent calculation. Unlike the Hartree contribution, it is not adiabatic, i.e., it depends on the retarded current, not the instantaneous density. Consider the one-dimensional example above cast in three dimensions: we fatten the ring in the radial and cylindrical directions, imposing either hard wall or periodic boundary conditions in these two directions. The current flows only azimuthally and uniformly on the ring and so is purely transverse in the sense that it has nonzero curl and zero divergence. At the same time, we call it macroscopic, since it is uniform along the ring. The classical response appears purely from $\mathbf{A}_{\mathrm{T}}$ rather than from Hartree. We have checked that the VK approximation for the exchange-correlation vector potential [12] is unaffected by the addition of $\mathbf{A}_{\mathrm{T}}$. (We note that the addition of $\mathbf{A}_{\mathrm{T}}$ to static current-density functional theory has been discussed for example in [27].)

We pause to make a connection with TDDFT. The TD density is given exactly in TDCDFT, via continuity $\dot{n}(\mathbf{r} t)=-\nabla \cdot \mathbf{j}(\mathbf{r} t)$ but a TDDFT calculation is only guaranteed to reproduce the longitudinal component of the current. If there does exist a TDDFT KS potential $v_{\mathrm{S}}(\mathbf{r} t)$ that reproduces the interacting current as well as the density [8], then the TDCDFT potential is $\mathbf{A}_{\mathrm{S}}(\mathbf{r} t)=c \int^{t} d t^{\prime} \nabla v_{\mathrm{S}}\left(\mathbf{r} t^{\prime}\right)$. But there exist special geometries, like the ring, to which TDDFT cannot be applied at all when macroscopic fields are present.

Next we consider the special case of linear response to a uniform electric field, $A_{\text {ext }}(\mathbf{r} t)=c \mathcal{E}(\omega) \exp (-i \omega t+$ $\eta t) / i \omega$, where $\eta=0_{+}$turns the field on adiabatically from $t=-\infty$ to 0 . If the current does not grow indefinitely (so that Fourier transforms exist), all additions to the external potential in Eq. (17) can be written to first order in the perturbation:

$$
\begin{aligned}
\mathbf{A}_{\mathrm{H}}(\mathbf{r} \omega) & =\frac{c}{(i \omega)^{2}} \int d^{3} r^{\prime} \nabla \frac{1}{\left|\mathbf{r}-\mathbf{r}^{\prime}\right|} \nabla^{\prime} \cdot \mathbf{j}\left(\mathbf{r}^{\prime} \omega\right) \\
\mathbf{A}_{\mathrm{T}}(\mathbf{r} \omega) & =\frac{1}{c} \int d^{3} r^{\prime} \frac{\exp \left(i \omega\left(\left|\mathbf{r}-\mathbf{r}^{\prime}\right| / c\right)\right) \mathbf{j}_{\mathrm{T}}\left(\mathbf{r}^{\prime} \omega\right)}{\left|\mathbf{r}-\mathbf{r}^{\prime}\right|} \\
\mathbf{A}_{\mathrm{XC}}(\mathbf{r} \omega) & =\int d^{3} r^{\prime} \overleftrightarrow{f}_{\mathrm{XC}}\left[n_{0}\right]\left(\mathbf{r r}^{\prime} \omega\right) \cdot \mathbf{j}\left(\mathbf{r}^{\prime} \omega\right)
\end{aligned}
$$

where $\stackrel{\leftrightarrow}{f}_{\text {XC }}$ is a nonlocal exchange-correlation tensor functional of the ground-state density, analogous to the scalar exchange-correlation kernel of TDDFT.

We return now to solids in time-varying electric fields, and consider the macroscopic response, using the ring geometry as described earlier. Let $\mathbf{G}$ be the reciprocal lattice vector. The density change when the external field is turned on

$$
\delta n(\mathbf{r} t)=\sum_{\mathbf{G} \neq 0} \delta n_{\mathbf{G}}(\omega) \exp (i \mathbf{G} \cdot \mathbf{r}-i \omega t)
$$

has no macroscopic (i.e., $\mathbf{G}=0$ ) component due to charge conservation. This implies that the Hartree response remains always periodic and has no macroscopic component. Performing a spatial Fourier transform on the transverse potential yields

$$
\mathbf{A}_{\mathrm{TG}}(\omega)=-\frac{4 \pi c}{\omega^{2}-c^{2} \mathrm{G}^{2}} \mathbf{j}_{\mathrm{TG}}(\omega) .
$$

We distinguish the microscopic $(\mathbf{G} \neq 0)$ transverse current from the macroscopic $(\mathbf{G}=0)$ current, which is a spatially uniform current travelling around the ring. As $\omega \rightarrow 0$, the microscopic contribution vanishes relative to Hartree, because of the factor of $\omega^{2}$ in Eq. (10). The macroscopic component, however, does not, yielding

$$
\begin{aligned}
& \mathbf{A}_{\mathrm{T}}^{\operatorname{mac}}(\omega)=\frac{4 \pi c}{(i \omega)^{2}} \mathbf{j}_{\mathbf{G}=0}(\omega) \\
& \mathbf{A}_{\mathrm{XC}}^{\operatorname{mac}}(\omega)=\sum_{\mathbf{G}} \stackrel{\leftrightarrow}{\mathrm{XC} 0 \mathbf{G}}^{\operatorname{ma}}\left[n_{0}\right](\omega) \cdot \mathbf{j}_{\mathbf{G}}(\omega) .
\end{aligned}
$$


Thus, from the ring perspective, the origin of the electromagnetic response (traditionally considered a Hartree effect) is the transverse potential generated by the ring current. In the limit when $L \rightarrow \infty$, the distinction between transverse and longitudinal breaks down, but for any real calculation with discrete $k$-points in the Brillouin zone, this distinction is important. For any $L, \mathbf{A}_{\mathrm{T}}^{\mathrm{mac}}(\omega)$, being the classical macroscopic response, cannot be neglected in considering solids in electric fields.

For insulators and metals at finite frequencies, after the adiabatic turn-on of the field, the system settles into a steady state, thanks to the damping factor $\eta$. Because we are using TDCDFT, the macroscopic current in this KS calculation is the physical one at all times. Thus the macroscopic polarization

$$
\mathbf{P}^{\mathrm{mac}}(t)=-\int_{-\infty}^{t} d t^{\prime} \mathbf{j}_{\mathbf{G}=0}\left(t^{\prime}\right), \quad \mathbf{P}^{\mathrm{mac}}(\omega)=\frac{\mathbf{j}_{\mathbf{G}=0}(\omega)}{i \omega}
$$

equals the physical one. Multiplying the vector potential by $i \omega / c$ yields the KS electric field:

$$
\begin{aligned}
\mathbf{E}_{\mathrm{xC}}^{\mathrm{mac}}(\omega)= & -\frac{\omega^{2}}{c} \overleftrightarrow{f}_{\mathrm{XC} 00}(\omega) \mathbf{P}^{\mathrm{mac}}(\omega)+\frac{i \omega}{c} \sum_{\mathbf{G} \neq 0} \\
& \overleftrightarrow{f}_{\mathrm{XC} 0 \mathbf{G}}(\omega) \cdot\left\{\frac{\mathbf{G} \omega n_{\mathrm{G}}(\omega)}{\mathrm{G}^{2}}+\mathbf{j}_{\mathrm{T} \mathbf{G}}(\omega)\right\}
\end{aligned}
$$

where we have used continuity. If the microscopic transverse currents vanish or can be neglected, then $\mathbf{j}_{\mathbf{G}}$ can be written solely in terms of $n_{\mathbf{G}}$, meaning that the entire corrections to the external field can be given in terms of the macroscopic polarization and the periodic density change. Now consider the final state of the system after the field has been adiabatically switched on. Then in the limit that $\omega \rightarrow 0$, this second point is precisely the GGG assertion made in density polarization functional theory: that the exchange-correlation field depends on both the periodic density and the macroscopic polarization [17, 18]. That result arises here out of TDCDFT, even at finite frequencies, and Eq. (15) then provides an exact expression for the polarization functional. However if microscopic transverse currents cannot be neglected, then for the GGG result to hold, these currents themselves would need to be functionals of the periodic density and macroscopic polarization; this remains to be investigated.

During the turning-on of the electric field, in the $\omega \rightarrow 0$ limit the system remains always in its adiabatic groundstate. Thus minimization of the energy (including the exchange-correlation electric field terms) over periodic functions 18, 29, 30], within the Berry-phase formalism [28, 30], will yield the same result as the TDCDFT calculation in the low-frequency limit.

This work also shows that polarization is an infinite memory effect within TDCDFT [31], i.e., after the electric field has reached a finite value, the system 'remembers' forever the current that flowed in turning it on. (It does not depend on the procedure in reaching the steadystate, rather it depends just on the time-integrated current). Similarly, in pumping a finite system from the ground-state to a given excited state, the current provides a natural way for the system to remember indefinitely which state it is in. Thus TDCDFT may provide a natural solution to some of the paradoxes generated by a pure time-dependent DFT 31].

Lastly, we discuss existing approximations and calculations. Just like the Hartree term, any density functional approximation, e.g. ALDA or AGGA, misses entirely the macroscopic contributions discussed here 33]. Even if we regard the bulk insulator as a large but finite slab, so that TDDFT does in principle apply, we would need an ultra-nonlocal functional of the density in order to capture the contribution to the $\mathrm{XC}$ field from the polarization charge density on the surfaces. In the static limit, this is (often) essentially the GGG effect discussed above, where in addition to the bulk periodic density, the functional depends on the bulk macroscopic polarization. For example, for a large rectangular slab with translation symmetry, the exchange-correlation electric field is

$$
\mathbf{E}_{\mathrm{XC}}(\mathbf{q}, \omega)=-i \mathbf{q} f_{\mathrm{XC}}(\mathbf{q}, \omega) n^{(1)}(\mathbf{q}, \omega)
$$

where the TDDFT kernel in the long wavelength limit may be expressed in terms of the component of the TDCDFT kernel parallel to the field: $\lim _{\mathbf{q} \rightarrow 0} f_{\mathrm{XC}}(\omega)=$ $-\omega^{2} \overleftrightarrow{f}_{\mathrm{xc} 00 \|}(\omega) /\left(c q^{2}\right)$. The ultranonlocal $1 / q^{2}$ dependence multiplies the response density, $n^{(1)}$ : this has a latticeperiodic part in the bulk as well as a part proportional to the polarization charge density on the surface. The fact that one needs to look only at a unit cell in the bulk and integrate up the current that has flowed through it to obtain $\mathbf{P}^{\mathrm{mac}}(t)$, shows how local approximations in terms of the current capture the essential physics, while local density approximations cannot. Since orbitals depend ultranonlocally on space, orbital functionals in TDDFT 32. may capture polarization effects. However, since polarization can be obtained from the current-density, these more complex functionals are not necessary to describe these effects if the current-density is used as the basic variable.

TDCDFT calculations for the optical response of solids have already been reported 13, 14] that explicitly include the macroscopic transverse component of $\mathbf{A}_{\mathrm{EM}}$ on top of the VK approximation. In adding the VK correction to their calculation 12], the microscopic contributions in Eq. (15) are not included (Eq. (16) of Ref. 14]). This may be related to the need to divide the beyond-ALDA contribution by a factor of 2.5. Similarly, the simple approximation in density polarization functional theory of Ref. [35], in which $\mathbf{E}_{\mathrm{xC}}=\gamma \mathbf{P}^{\mathrm{mac}}$, where $\gamma$ is a constant, does not include such contributions.

We conclude by noting that while the present work has focussed on the special case of the interior of a bulk insulator or metal, the underlying logic was motivated by the need to construct an approximately local theory of 
exchange-correlation for any system in an electric field. Two examples make this clear. In tunnelling through a quantum wire, present calculations use ground-state DFT KS orbitals as their starting point. This leads to resonances at the positions of bare KS orbitals. For finite systems without currents, regular DFT tells us there are significant exchange-correlation corrections. The only way to calculate such corrections for a quantum wire is using TDCDFT, in order to handle currents. In another area, electron and energy transfer in biological molecules, attempts are being made to estimate matrix elements in a TDDFT calculation 36]. Such calculations, using adiabatic local and gradient-corrected approximations, clearly miss any contributions from macroscopic currents.

This work was inspired by that of Profs. Kohn and Tolkien, and supported by the Office of Naval Research under grant no. NOOOO14-01-1-1061. We thank David Vanderbilt, Ralph Gebauer, Roberto Car, Hardy Gross, and Giovanni Vignale for useful discussions.
[1] P. Hohenberg and W. Kohn, Phys. Rev. 136, B 864 (1964).

[2] W. Kohn and L.J. Sham, Phys. Rev. 140, A 1133 (1965).

[3] R.O. Jones and O. Gunnarsson, Rev. Mod. Phys. 61, 689 (1989).

[4] W. Kohn, Rev. Mod. Phys. 71, 1253 (1999).

[5] E. Runge and E.K.U. Gross, Phys. Rev. Lett. 52, 997 (1984).

[6] M. Petersilka, U.J. Gossmann, and E.K.U. Gross, Phys. Rev. Lett. 76, 1212 (1996).

[7] M.E. Casida, in Recent developments and applications in density functional theory, ed. J.M. Seminario (Elsevier, Amsterdam, 1996).

[8] N. T. Maitra, K. Burke, H. Appel, E.K.U. Gross, and R. van Leeuwen, in Reviews in Modern Quantum Chemistry: A celebration of the contributions of R. G. Parr, ed. K. D. Sen, (World-Scientific, 2002).

[9] S. Albrecht, L. Reining, R. Del Sole, and G. Onida, Phys. Rev. Lett. 40, 4510 (1998).

[10] Y-H. Kim and A. Görling, Phys. Rev. Lett. 89, 096402 (2002).

[11] L. Reining, V. Olevano, A. Rubio and G. Onida, Phys. Rev. Lett. 88, 066404 (2002).

[12] G. Vignale and W. Kohn, Phys. Rev. Lett. 77, 2037 (1996).

[13] F. Kootstra, P.L. Boeij, and J.G. Snijders, J. Chem. Phys. 112, 6517 (2000).

[14] P.L. de Boeij, F. Kootstra, J.A. Berger, R. van Leeuwen, and J.G. Snijders, J. Chem. Phys. 115, 1995 (2001).

[15] M. van Faassen, P. L. de Boeij, R. van Leeuwen, J. A. Berger, and J. G. Snijders, Phys. Rev. Lett. 88, 186401 (2002).

[16] J. Autschbach and T. Ziegler, J. Chem. Phys. 116, 891 (2002).

[17] X. Gonze, Ph. Ghosez, and R.W. Godby, Phys. Rev. Lett. 74, 4035 (1995).

[18] R.M. Martin and G. Ortiz, Phys. Rev. B. 56, 1124 (1997).

[19] W. Kohn, Phys. Rev. 133, A171 (1964).
[20] B-X. Xu and A. K. Rajagopal, Phys. Rev. A. 31, 2682 (1985).

[21] E.K.U. Gross and W. Kohn, in Advances in Quantum Chemistry, Vol. 21: Density Functional Theory of ManyFermion Systems, edited by S. B. Trickey (Academic Press, San Diego, 1990).

[22] S.K. Ghosh and A.K. Dhara, Phys. Rev. A 38, 1149 (1988).

[23] T.K. Ng, Phys. Rev. Lett. 62, 2417, (1989).

[24] The proof of Ghosh and Dhara [22] chooses a gauge in which the scalar potential is not zero, but this is not necessary. See also E.K.U. Gross, J.F. Dobson, and M. Petersilka, in Topics in Current Chemistry, 181, 81 (1996).

[25] Ambegaokar and W. Kohn, Phys. Rev. 117, 423 (1960); R.M. Pick, M.H. Cohen, and R.M. Martin, Phys. Rev. B. 1, 910 (1970).

[26] K. Kunc and R. Resta, Phys. Rev. Lett. 51, 686 (1983).

[27] G. Vignale and M. Rasolt, Phys. Rev. B. 37, 10685 (1987).

[28] R. D. King-Smith and D. Vanderbilt, Phys. Rev. B. 47, 1651 (1993).

[29] R.W. Nunes and X. Gonze, Phys. Rev. B. 63, 155107 (2001).

[30] R. W. Nunes and D. Vanderbilt, Phys. Rev. Lett. 73, 712 (1994).

[31] N. T. Maitra, K. Burke, and C. Woodward, Phys. Rev. Lett. 89, 023002, (2002).

[32] C.A. Ullrich, U.J. Gossman, and E.K.U. Gross, Phys. Rev. Lett. 74, 872 (1995).

[33] G.F. Bertsch, J-I. Iwata, A. Rubio, and K. Yabana, Phys. Rev. B. 62, 7998 (2000).

[34] G. Vignale, C. A. Ullrich, and S. Conti, Phys. Rev. Lett. 79, 4878 (1997).

[35] W.G. Aulbur, L. Jönsson and J. W. Wilkins, Phys. Rev. B 54, 8540 (1996).

[36] R. Cave, K. Burke, and E. W. Castner Jr., J. Phys. Chem. A 106, 9294 (2002). 